\title{
Determination of the components of the gyration tensor of quartz by oblique incidence transmission two-modulator generalized ellipsometry
}

\author{
Oriol Arteaga, ${ }^{1, *}$ Adolf Canillas, ${ }^{1}$ and Gerald E. Jellison, Jr. ${ }^{2}$ \\ ${ }^{1}$ FEMAN Group, Departament de Física Aplicada i Òptica, IN2UB, \\ Universitat de Barcelona, Barcelona 08028, Spain \\ 2Oak Ridge National Laboratory, Oak Ridge, Tennessee 37830, USA \\ ${ }^{*}$ Corresponding author: oarteaga @ub.edu
}

Received 31 July 2009; accepted 14 August 2009;

posted 3 September 2009 (Doc. ID 115074); published 21 September 2009

\begin{abstract}
The two independent components of the gyration tensor of quartz, $g_{11}$ and $g_{33}$, have been spectroscopically measured using a transmission two-modulator generalized ellipsometer. The method is used to determine the optical activity in crystals in directions other than the optic axis, where the linear birefringence is much larger than the optical activity. () 2009 Optical Society of America

OCIS codes: $\quad 260.1180,260.1440,160.1585,240.2130$.
\end{abstract}

\section{Introduction}

Optical activity is a property of materials related to different refractive indices for right- and leftcircularly polarized light. Mathematically, optical activity is expressed as a complex frequency-dependent function, where the real dispersive part and the imaginary absorptive part are related through the Kramers-Kronig relation. The dispersive part is referred to as circular birefringence (CB), optical rotatory power (ORP), or optical rotatory dispersion (ORD), whereas the absorptive part is referred to as circular dichroism (CD).

The optical activity may be derived from a chiral packing arrangement of the constituent molecules or from the chirality of the individual molecules. In solid crystals the optical activity is normally associated with the absence of a center of symmetry in the crystal structure, because the constituent molecules or atoms of crystals are not generally chiral. However, the optical activity in liquids is usually

0003-6935/09/285307-11\$15.00/0

(C) 2009 Optical Society of America due to the chirality of the individual molecules. As a result, solid crystals tend to show much higher CB than liquids.

Optical activity was first observed by Arago [1] and Biot [2] in the early 1800s. The determination of CB has been subject of extensive experimental study, particularly for many crystals. Alpha-quartz $\left(\mathrm{SiO}_{2}\right)$ is probably the most studied, where the parameters of optical activity are known with great accuracy $[3,4]$, as well as the variations with temperature and pressure $[5,6]$. As a result, quartz plates are routinely used as a standard of calibration for commercial polarimeters. Most of the current measurements of $\mathrm{CB}$ are performed along the unique direction in the crystal (the $c$ axis for quartz), which has no linear birefringence, isolating the $\mathrm{CB}$. Therefore, most of the commercial quartz crystals are cut perpendicular to the $c$ axis.

Although both linear birefringence and optical activity have been separately studied using a variety of techniques, very few attempts have been made to quantify the optical activity in the presence of linear birefringence. For transparent materials, an accurate measurement of $\mathrm{CB}$ cannot be made without 
a full account of the related linear birefringence; for absorbent materials, the linear dichroism and CD must also be taken into account. In nature, circular and linear birefringent effects often appear together, so a method to measure CB in the presence of linear birefringence is highly desirable. One such measurement technique is the high-accuracy universal polarimeter (HAUP) [7-11]. This technique is a variation of the old technique of crossed polarizers, and it involves making measurements at slightly deviated angles from the position of maximum extinction of the polarizers. Typically, a monochromatic light source is used and the values of the optical activity are determined from the evolution of the detected waveform as a function of the angle of rotation of the polarizers.

The experimental approach we present is based on the use of photoelastic modulators (PEMs). For years, accurate measurements of birefringence have been made by using PEMs that dynamically elliptically polarize the input light $[12,13]$. PEMs are also employed in CD spectrometers [14,15]. However, most of the reported techniques that use PEMs, although very accurate, only measure a few elements of the sample Mueller matrix (in some cases, only one). One technique that can be used to measure the complete sample Mueller matrix is the twomodulator generalized ellipsometer (2-MGE) $[16,17]$. This powerful instrument uses two PEMs to completely measure the polarization-dependent optical properties of any sample in reflection or transmission, and has already been used to study the optical activity of organic materials [18].

In this paper, we present a method to measure the optical activity of crystals using the 2-MGE technique. Using this technique, it is possible to perform measurements in directions other than along the optic axis of the crystal, where the magnitude of the linear birefringence is significantly larger than the magnitude of the optical activity. As an application of this method we have measured the components of the gyration tensor of a right-handed $(\mathrm{RH})$ and a left-handed (LH) quartz crystal.

\section{Experimental}

The 2-MGE was introduced by Jellison and Modine in $1997[16,17]$. In subsequent years the technique was used in transmission [19] and normal-incidence reflection configurations [20], both of which can be used to obtain $x-y$ plots of the relevant parameters. The measurements presented herein were made with a spectroscopic 2-MGE with a variable angle of incidence that is also capable of operating in transmission.

The 2-MGE is composed of two polarizer-PEM pairs, each of the PEMs operating at a different resonant frequency (50 and $60 \mathrm{kHz}$ in this case), which is essential for frequency analysis. The first polarizer-PEM pair is used as the polarization state generator (PSG) on the input arm of the ellipsometer, while the second is used as the polarization state analyzer (PSA) on the detection arm of the ellipsometer. A PEM is a resonant device that is cut to oscillate at a determinate frequency and acts as an optical element with a time-dependent retardation. Both the operating frequency and the amplitude of modulation are very stable but, while the amplitude of modulation can be easily controlled (usually with an external dc voltage), the frequency of oscillation (typically at $20-80 \mathrm{kHz}$ ) is given by the geometry of the resonant bar.

A schematic of the optical train of the experimental setup is given in Fig. 1. The light source is a $75 \mathrm{~W}$ xenon arc lamp (Oriel) that is focused onto a quartz optical fiber with a $1000 \mu \mathrm{m}$ core. The end of the optical fiber is connected to a mirror-based focusing system (Jobin-Yvon), resulting in a spot size from $2 \mathrm{~mm}$ to $100 \mu \mathrm{m}$ at the sample. The PSG is comprised of a Rochon $\mathrm{MgF}_{2}$ polarizer and a PEM (Hinds Instruments) oscillating at $50 \mathrm{kHz}$. The linear polarizer is attached to the PEM using a precision manual rotator and is oriented at $45^{\circ}$ with respect to the PEM orientation. The PSG is attached to a stepper rotation stage (National Instruments UE31PP) so that different incoming polarization states can be incident upon the sample.

The PSA has the same components as the PSG except for the operation frequency of the PEM, which, in this case, has a nominal frequency of $60 \mathrm{kHz}$. The PSA is also mounted on an automated rotator so that different polarization states coming from the sample can be analyzed.

The light beam passing through the PSA is focused onto a circular-to-rectangular quartz fiber bundle, which guides the light to the double grating monochromator (Jobin-Yvon DH10). The rectangular $(200 \mu \mathrm{m} \times 6 \mathrm{~mm})$ end of the fiber acts as entrance slit of the monochromator. The light intensity is detected at the output slit of the monochromator by using a photomultiplier tube (PMT) (Hamamatsu R3896). The signal from the PMT is in the form of a photocurrent and is converted to a voltage by using a current-mode preamplifier (Hamamatsu C7319). The voltage waveform from the preamplifier is captured by using a computer digitizer board (Spectrum MI 3130) which is configured to capture 16,384 samples in $8 \mathrm{~ms}$. As there are two modulators running free, each of them with its own phase, there is a trigger circuit that initializes the data capture when the

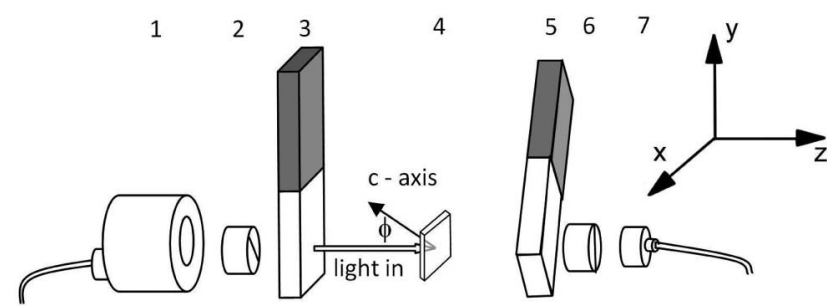

Fig. 1. Diagram of the experimental setup: 1, focusing system; 2, polarizer; 3, PEM $0(50 \mathrm{kHz}) ; 4$, sample (tilted quartz plate); 5, PEM $1(60 \mathrm{kHz}) ; 6$, analyzer; 7, lens. Light collected in 7 is guided by an optical fiber to a monochromator. 
two modulators are in phase. The computer board includes programmable analog outputs that are used to control the modulation amplitude of the PEMs, which is kept at $A_{0}=A_{1}=2.4048 \mathrm{rad}$ [so that $J_{0}\left(A_{0}\right) \simeq J_{0}\left(A_{1}\right) \simeq 0$ ], and the gain introduced by the PMT, so that the dc component of the signal is kept constant during measurement.

The 2-MGE technique preserves the benefits of conventional phase-modulated ellipsometry with the advantage that is capable of measuring eight normalized Mueller matrix elements simultaneously. In contrast, the ellipsometers with only one photoelastic modulator are limited to measuring only two elements simultaneously. However, the interpretation of the 2-MGE waveform is more complicated, because at least eight frequency components have to be determined by Fourier analysis in one single measurement. For this work, the magnitude and phase of 12 frequency components are determined in one single measurement, resulting in four of the eight parameters being measured twice. A detailed description of the Fourier integral analysis of the time-dependent detected intensity is given in [16].

For most situations in which there is no depolarization, a single measurement of 2-MGE is sufficient to completely determine the polarization-dependent optical properties of a sample that could be described with a Mueller-Jones matrix. For a depolarizing sample, all the elements of the normalized Mueller matrix can be determined with four measurements at different orientations of the azimuthal angles of the PSG and the PSA. Namely, these four configurations (PSG, PSA) are $\left(0^{\circ}, 0^{\circ}\right),\left(0^{\circ}, 45^{\circ}\right),\left(45^{\circ}, 0^{\circ}\right)$, and $\left(45^{\circ}, 45^{\circ}\right)$. In each one of these configurations, eight elements of the normalized Mueller matrix are determined; therefore, some of the elements are overdetermined (for example, the elements $m_{03}, m_{30}$, and $m_{33}$ are measured four times) and, when this happens, the final values for these particular elements are the averaged values.

The geometry of the problem is shown in Fig. 2 , where the sample is quartz crystal with the $c$ axis perpendicular to the optical faces of the sample. A light beam is incident upon the crystal at an angle

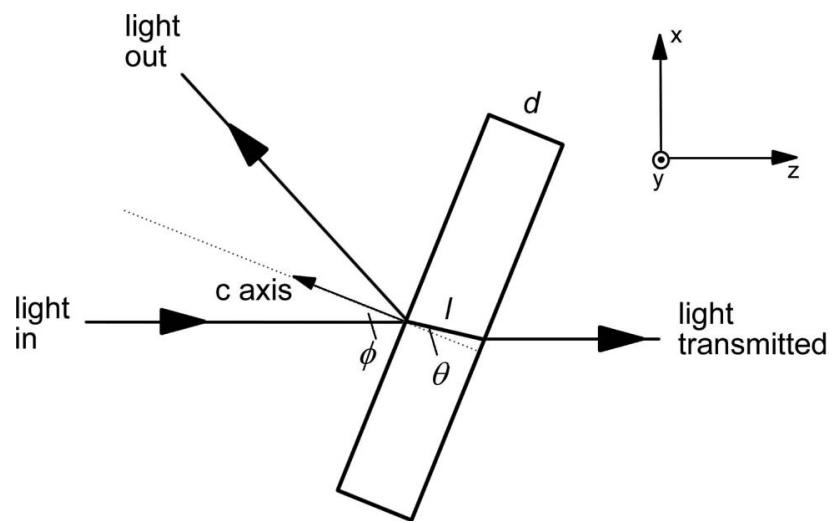

Fig. 2. Geometry of the off-normal-incidence measurement of circular and linear birefringence. of incidence $\phi$ with respect to its $c$ axis. $l, d$, and $\theta$ are, respectively, the light path through the crystal, the sample thickness, and the angle with respect to the $c$ axis of the wave normal for the wave propagating through the crystal. If $d$ and $\phi$ are known, the light path $l$ can be found by taking the approximation that the wave normal (given by Snell's law) in the crystal coincides with the direction of propagation:

$$
l=\frac{d}{\cos \theta}, \quad \theta=\arcsin \left(\frac{\sin \phi}{\bar{n}}\right),
$$

where $\bar{n}$ is the mean refractive index, which depends on $\theta$ and will be defined in Section 3 . The sample was tilted in order to perform measurements at different values for $\phi$ (which also means different values for $\theta$ ). The rotation was around the $y$ axis defined by the modulation axis of the PEMs when PSG and PSA are orientated at 0 . A general scheme of the sample positioning in the instrument is also shown in Fig. 1.

The various birefringence parameters are defined as follows:

$$
\begin{aligned}
\mathrm{LB} & =\frac{2 \pi}{\lambda}\left(n_{x}-n_{y}\right) l, \\
\mathrm{LB}^{\prime} & =\frac{2 \pi}{\lambda}\left(n_{45}-n_{135}\right) l \\
\mathrm{CB} & =\frac{2 \pi}{\lambda}\left(n_{-}-n_{+}\right) l,
\end{aligned}
$$

where $\lambda$ is the wavelength of light and the subscripts specify the polarization of light as $x, y, 45^{\circ}$ to the $x$ axis, $135^{\circ}$ to the $x$ axis, circular right + , or left - . The circular birefringence is twice the optical rotation. The $x-y$ linear birefringence LB is very sensitive to sample tilt around the $y$ axis, and so will be much more sensitive than the $45^{\circ} \mathrm{LB}^{\prime}$, which is expected to be small.

The samples studied in this paper consist of two $z$ cut ( $c$ axis perpendicular to sample surface) quartz crystals $\left(\mathrm{SiO}_{2}\right)$ with opposite handedness. Crystalline quartz (point group 32) is enantiomorphous, in that it may occur as either right- or left-handed. In order to be enantiomorphous, a crystal must have no element of symmetry that changes the handedness. For quartz, the atomic arrangement provides a screw axis that produces a helical distribution of atoms, and the optical activity of the two types of handedness has opposite signs. We will present results separately for the $\mathrm{LH}$ plate and for the $\mathrm{RH}$ plate. The measured thickness of the LH plate is $1.02 \pm 0.01 \mathrm{~mm}$, while the thickness of the $\mathrm{RH}$ plate is $1.06 \pm 0.01 \mathrm{~mm}$.

In the case of quartz crystal, the CB is known to be nonzero in a wide frequency region that includes the whole studied spectrum (220 to $800 \mathrm{~nm}$ ) [21]. In this region quartz is not absorbent [22] and, therefore, all parameters related to the absorptive nature of the crystal (including the CD) are zero.

\section{Theory}

The theory of light propagation in general anisotropic media often results in complicated equations. For 
optical active crystals where the direction of light propagation is along the optical axis, the problem can be treated with the phenomenological equations that are analogous to those used in isotropic systems, resulting in significant simplification. However, the more general case of oblique incidence requires solving the Maxwell equations with modified constitutive equations, significantly complicating the mathematics. To do so, several authors use the Berreman $4 \times 4$ matrix formulation of Maxwell equations, but, even so, different approaches appear in the literature since the choice of the constitutive relations is not unique [23]. We will use the propagation description first given by [24], which uses the constitutive equations given by Born [25]. This model is widely used in the literature [23,26-28], particularly in experimental work, and gives the possible values of the refractive index $n$, for a given direction of the wave normal. Although this approach is only approximate, it has been shown to be accurate as long as the birefringence of the investigated crystal is not huge [29], as this model describes the phenomenon of optical activity within an accuracy of the product of the linear birefringence in the direction of observation by the gyrotropy parameters [28]. In this context, the two possible refractive indices are given by the positive roots of the equation $[\underline{27,28]}$

$$
\left(n^{2}-n_{01}^{2}\right)\left(n^{2}-n_{02}^{2}\right)=G^{2},
$$

where $n_{01}$ and $n_{02}$ are the refractive indices of the eigenwaves in the absence of optical activity. $G$ is the scalar gyration parameter and is a measure of the optical activity for the direction in question. $G$ as a function of direction is given by

$$
G=g_{i j} l_{i} l_{j}
$$

in which the convention of summing over repeated indices is used. $l_{i}$ and $l_{j}$ are the direction cosines of the normal wave and $g_{i j}$ are the components of the gyration tensor that describe the optical activity of the crystal. In uniaxial crystals $\left(g_{11}=g_{22} \neq g_{33}\right) G$ takes the form [23]

$$
G=g_{11} \sin ^{2} \theta+g_{33} \cos ^{2} \theta,
$$

where $\theta$ is defined in Eq. (1).

The solutions of Eq. (3) are [23]

$$
n_{1,2}^{2}=\left[n_{01}^{2}+n_{02}^{2} \mp\left[\left(n_{02}^{2}-n_{01}^{2}\right)^{2}+4 G^{2}\right]^{1 / 2}\right] / 2,
$$

and, for a uniaxial crystal $\left(\varepsilon_{11}=\varepsilon_{22} \neq \varepsilon_{33}\right)$,

$$
n_{01}^{2}=\varepsilon_{11}, \quad n_{02}^{2}=\frac{\varepsilon_{11} \varepsilon_{33}}{\left(\varepsilon_{11} \sin ^{2} \theta+\varepsilon_{33} \cos ^{2} \theta\right)},
$$

where $\varepsilon_{11}=\varepsilon_{o}=n_{o}^{2}$ and $\varepsilon_{33}=\varepsilon_{e}=n_{e}^{2}$. The refractive indices $n_{o}$ and $n_{e}$ of quartz are well known and are given by the five-parameter dispersion relation calculated in [30].
The total birefringence $\left(T_{R}\right)$ of the system is a combination of linear (LB and $\left.\mathrm{LB}^{\prime}\right)$ and circular (CB) birefringences:

$$
T_{R}^{2}=\mathrm{LB}^{2}+\mathrm{LB}^{2}+\mathrm{CB}^{2},
$$

with

$$
T_{R}=\frac{2 \pi}{\lambda}\left(n_{2}-n_{1}\right) l
$$

and LB, $\mathrm{LB}^{\prime}$, and $\mathrm{CB}$ as defined in Eq. (2). According to Eq. (6) and assuming that $G^{2} \ll n_{01}^{2} n_{02}^{\overline{2}}, T_{R}^{2}$ can be approximated by

$$
\mathrm{T}_{R}^{2} \simeq \frac{4 \pi^{2}}{\lambda^{2}}\left[\left(n_{02}-n_{01}\right)^{2}+\frac{G^{2}}{n_{01} n_{02}}\right] l^{2} .
$$

Because of the geometry of our oblique incidence experiment (see Fig. 2), the sample tilting does not cause birefringence in direction $45^{\circ}$ to the $x$ axis $\left(\mathrm{LB}^{\prime} \simeq 0\right)$ and $\mathrm{LB}$ itself can be considered as a measure of the total linear birefringence. Therefore, Eq. (8) can be simplified to

$$
\mathrm{T}_{R}^{2} \simeq \mathrm{LB}^{2}+\mathrm{CB}^{2} .
$$

Comparing Eq. (11) to Eqs. (10) and (ㅇ), we can make the following identifications,

$$
\mathrm{LB}=\frac{2 \pi}{\lambda}\left(n_{02}-n_{01}\right) l,
$$

$$
\mathrm{CB}=\frac{2 \pi G l}{\lambda \bar{n}},
$$

where $\bar{n}$ stands for a mean refractive index $\bar{n}=\sqrt{n_{01} n_{02}}$.

In the special case of propagation along the optic axis $\left(\theta=0^{\circ}\right) \mathrm{CB}$ is proportional to the $g_{33}$ component of the gyration tensor:

$$
g_{33}=\frac{\mathrm{CB}_{\theta=0^{\circ}} \lambda n_{o}}{2 \pi l} .
$$

For light propagation along the optical axis in an optically active uniaxial crystal or for light propagation in any direction in an optically active isotropic medium, the eigenmodes of optical propagation through the material are left and right circularly polarized modes, each of which has its own refractive index. In contrast, the eigenmodes in a uniaxial material without optical activity are linearly polarized modes that are mutually orthogonal. As a result, in a random direction in an optically active uniaxial crystal, the eigenmodes are left and right elliptical polarization modes with major axes that coincide with the directions of the linearly polarized 
eigenmodes that would be present in the absence of optical activity.

The ellipticity $k$ is the ratio of the minor axis to the major axis of the ellipse of polarization, and it can be used to define the waves that travel through the crystal unchanged in form. Along the optic axis, $k=1$, which indicates that the eigenmodes are circularly polarized light. The quantity $k$ can be calculated as follows [27]:

$$
k=\tan \left(\frac{\gamma}{2}\right),
$$

where $\gamma$ is an angle defined by the ratio between the circular and linear birefringences:

$$
\tan \gamma=\frac{\mathrm{CB}}{\mathrm{LB}} .
$$

With the relations of Eqs. (12) and (13), this ratio can be written as

$$
\frac{\mathrm{CB}}{\mathrm{LB}}=\frac{G}{\bar{n}\left(n_{02}-n_{01}\right)},
$$

and, by substituting Eq. (5) here, we can derive an expression to calculate $g_{11}$ from experimental measurements of the ratio $\mathrm{CB} / \mathrm{LB}$ :

$$
g_{11}=\frac{1}{\sin ^{2} \theta}\left[\bar{n}\left(n_{02}-n_{01}\right) \frac{\mathrm{CB}}{\mathrm{LB}}-g_{33} \cos ^{2} \theta\right] .
$$

\section{A. Model for the Dispersion of the Gyration Tensor Components}

The first phenomenological models to describe the optical activity appeared early in the 20th century [3,31], where the first tenuous connections were made between optical rotation and the interaction between atoms. One of the more successful ap-

$$
\rho=\frac{k \lambda^{2}}{\left(\lambda^{2}-\lambda_{0}^{2}\right)^{2}}
$$

The optical rotation $\rho$ is related to $\mathrm{CB}$ by $\rho=1 / 2 \mathrm{CB}$, and $k$ and $\lambda_{0}$ are the two unique parameters of the model.

To measure the components of the gyration tensor $G$, we propose to use this dispersion model to parameterize $g_{11}$ and $g_{33}$ :

$$
g_{i i}=\frac{A_{i} \lambda^{3}}{\left(\lambda^{2}-B_{i}^{2}\right)^{2}}
$$

where $A_{i}$ and $B_{i}$ are the parameters to be determined. Note that this model can be straightforwardly derived from Eq. (19) if we consider that $G=\lambda \bar{n} \rho / \pi l$ [see Eq. (13)] and we use the approximation that $\bar{n}$ is constant. We have found that Eq. (20) is a good fit for our experimental $g_{11}$ and $g_{33}$ data. It is interesting to note that, while for quartz, $\mathrm{CB}\left(\propto\left(n_{-}-n_{+}\right)\right)$varies by more than 1 order of magnitude in the interval range from 200 to $800 \mathrm{~nm}$, the variation of the mean refractive index $\left.\bar{n}\left(\propto\left(n_{-}+n_{+}\right)\right)\right)$in this same interval is only around $7 \%$. Thus, it is not strange that, if Eq. (19) constitutes a good fit for CB, Eq. (20) can be used to fit the gyration tensor with success.

\section{Data Analysis for the Mueller Matrix of Quartz}

The spectroscopic Mueller matrices measured at different small incident angles for $\mathrm{RH}$ and $\mathrm{LH}$ plates are, respectively, presented in Figs. 3 and 4 . In both cases, and for all the orientations of the plates, Mueller matrix elements $m_{01}, m_{02}, m_{03}, m_{10}, m_{20}$, and $m_{30}$ are approximately zero in all the studied spectral range (note that these elements have been multiplied by a factor 10). This indicates that, at this wavelength range and for these small angles of incidence, there is not significant diattenuation and the Mueller matrices of the quartz plates basically take the form of a general retarder [33]:

$$
\mathbf{M}_{R}=\left(\begin{array}{cccc}
1 & 0 & 0 & 0 \\
0 & \cos T_{R}+\mathrm{LB}^{2} \alpha & \mathrm{LBLB}^{\prime} \alpha+\mathrm{CB} \beta & -\mathrm{LBCB} \alpha+\mathrm{LB}^{\prime} \beta \\
0 & \mathrm{LBLB}^{\prime} \alpha-\mathrm{CB} \beta & \cos T_{R}+\mathrm{LB}^{\prime 2} \alpha & -\mathrm{LB}^{\prime} \mathrm{CB} \alpha-\mathrm{LB} \beta \\
0 & -\mathrm{LBCB} \alpha-\mathrm{LB}^{\prime} \beta & -\mathrm{LB}^{\prime} \mathrm{CB} \alpha+\mathrm{LB} \beta & \cos T_{R}+\mathrm{CB}^{2} \alpha
\end{array}\right)
$$

proaches was carried out by Chandrasekhar using classical theory to study the optical rotation of quartz $[21,32]$. In this model there are two coupled oscillators that represent the smallest unit of the optically active crystal. The oscillators are assumed to be identical and undamped (that is, the crystal is nonabsorbing). This coupled-oscillator model can also be treated quantum mechanically and describes the optical rotation $\rho$ of quartz in a wide frequency region: with $\quad \alpha=\left(1-\cos T_{R}\right) / T_{R}^{2}, \quad \beta=\sin T_{R} / T_{R}, \quad$ and $T_{R}=\sqrt{\mathrm{LB}^{2}+\mathrm{LB}^{2}+\mathrm{CB}^{2}}$.

However, a crystal with retardation properties that vary rapidly with wavelength may induce depolarization if the system collects a band of wavelengths rather than a single wavelength. Since the retardation is proportional to the inverse of the wavelength, a band of wavelengths will result in a 


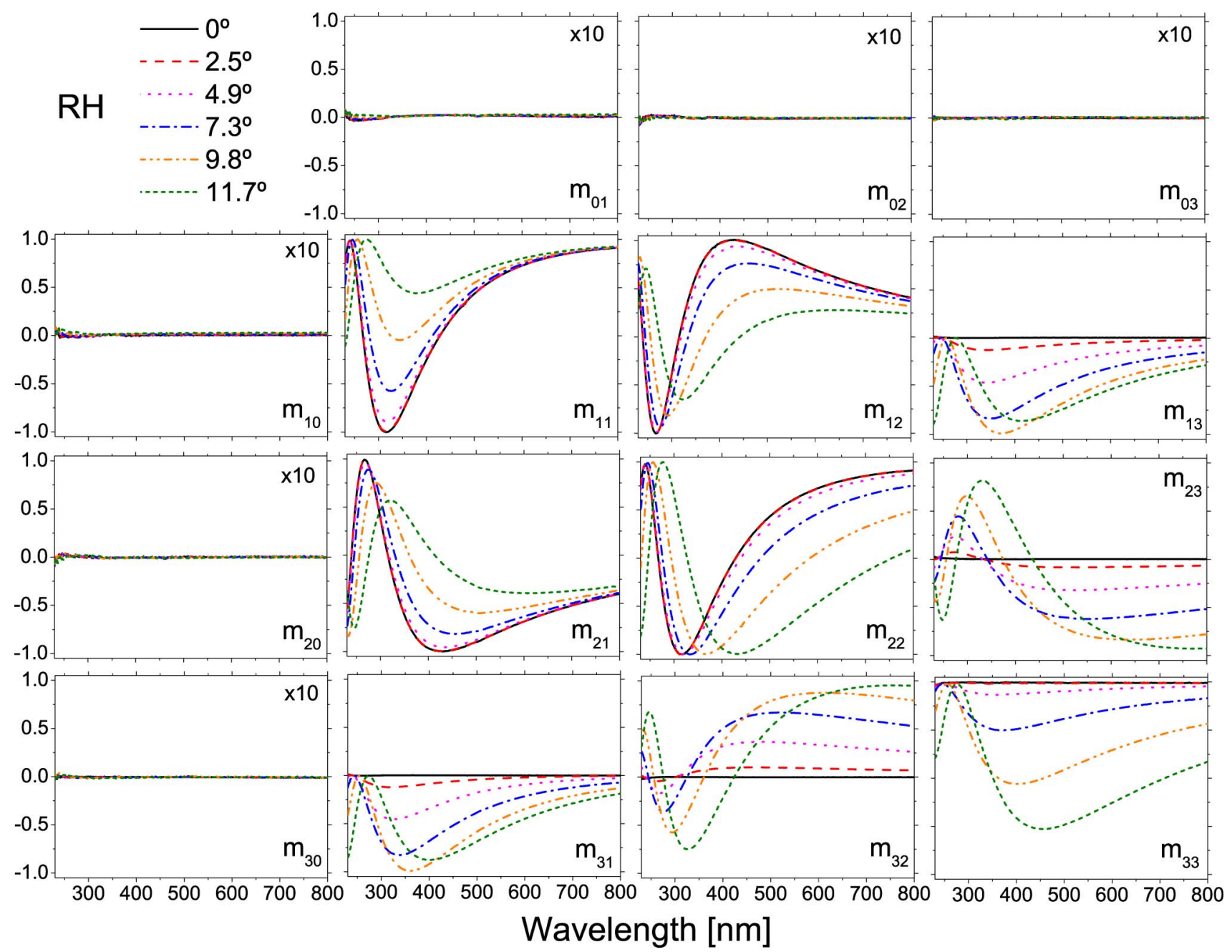

Fig. 3. (Color online) Spectroscopic Mueller matrix for a RH plate of quartz at small incidence angles $(\phi)$.

different retardation for each wavelength, resulting in partially polarized light [34]. To minimize this depolarization, the monochromator slit width can be reduced and/or a smaller core optical fiber can be used at the input of the monochromator [19]. The fraction of polarized light $\beta$ in the light beam can be calculated from the Mueller matrix as follows [35]:

$$
\beta=\frac{\sqrt{\left(\sum_{i j} m_{i j}^{2}\right)-m_{00}^{2}}}{\sqrt{3} m_{00}},
$$

where $\beta=1$ for totally polarized light and $\beta=0$ for a completely unpolarized beam. Figure 5 depicts a comparison between the fraction of polarized at normal incidence and at $60^{\circ}$ incidence. As can be seen in the figure, the depolarization becomes particularly significant for big angles of incidence, where the observed retardation has an important variation with wavelength, and it needs to be considered.

To consider the depolarization of the measured Mueller matrices, we have factorized them according to the polar decomposition [36]. Briefly, this decomposition states that a Mueller matrix M can be factored into three matrices:

$$
\mathbf{M}=\mathbf{M}_{\Delta} \mathbf{M}_{R} \mathbf{M}_{D}
$$

where $\mathbf{M}_{D}$ is a diattenuator, $\mathbf{M}_{R}$ is a retarder, and $\mathbf{M}_{\Delta}$ is a depolarizer. This factorization allows the separation of the depolarization properties from the retardation and dichroic properties. For a uniaxial crystal, the Mueller matrices $\mathbf{M}_{R}$ and $\mathbf{M}_{D}$ commute [33], so the polar decomposition can be used to factor the experimental Mueller matrix. Once the retardation matrix $\mathbf{M}_{R}$ has been calculated, the values of linear and circular birefringence can be extracted as follows:

$$
\begin{aligned}
T_{R} & =\arccos \left(\operatorname{tr}\left(\mathbf{M}_{R}\right) / 2-1\right), \\
\mathrm{LB} & =\left(m_{R_{32}}-m_{R_{23}}\right) \frac{T_{R}}{2 \sin T_{R}}, \\
\mathrm{LB}^{\prime} & =\left(m_{R_{13}}-m_{R_{31}}\right) \frac{T_{R}}{2 \sin T_{R}}, \\
\mathrm{CB} & =\left(m_{R_{12}}-m_{R_{21}}\right) \frac{T_{R}}{2 \sin T_{R}},
\end{aligned}
$$

where the term $m_{R_{i j}}$ indicates matrix elements of the $i$ th row and the $j$ th column of $\mathbf{M}_{R}$.

When dealing with samples that introduce significant retardations, such as crystals, the 


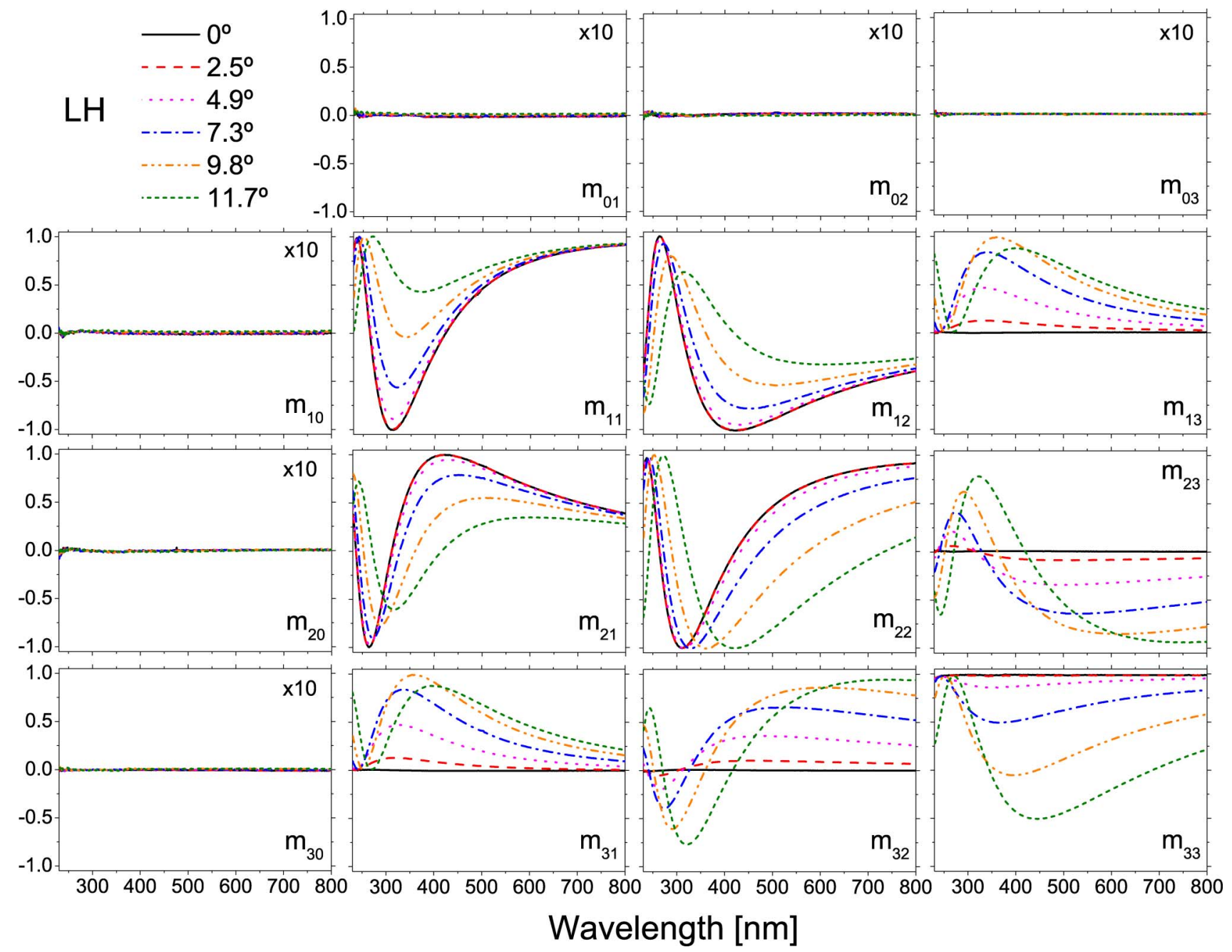

Fig. 4. (Color online) Spectroscopic Mueller matrix for a LH plate of quartz at small incidence angles $(\phi)$.

determination of the sample Mueller matrix may not be sufficient to determine all its birefringent characteristics. In Eq. (21), it can be seen that many of the Mueller matrix elements contain trigonometric functions. Furthermore, the arccosine, which is multivalued, is required to determine the total birefringence [see Eq. (24)]. For systems with small retardations,

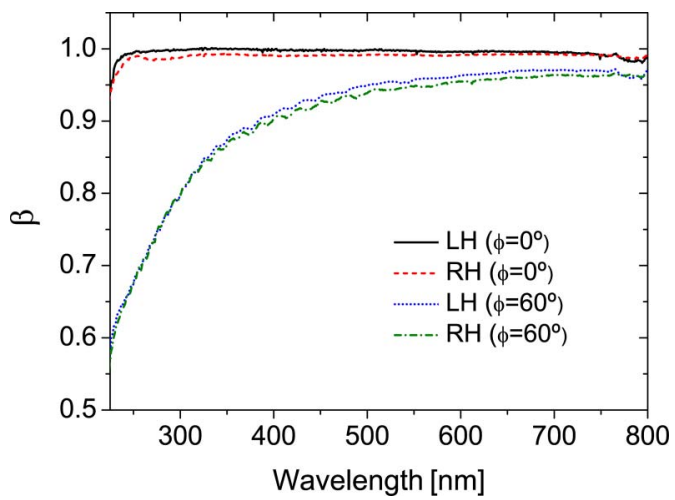

Fig. 5. (Color online) Fraction $\beta$ of polarized light for normal and oblique incidence. For the same conditions, the RH plate is a bit more depolarizing because it is a slightly thicker than the $\mathrm{LH}$ plate. this does not constitute a problem since the total retardation must fall in the $[0, \pi]$ interval. However, for systems with big retardances (e.g., some crystals), this problem prevents us from determining the birefringences unless we know their order. For example, at $252 \mathrm{~nm}$, the Mueller matrix of the $\mathrm{LH}$ plate of quartz with an angle of incidence of $9^{\circ}$ is the following:

$$
\mathbf{M}=\left[\begin{array}{cccc}
1 & 0.002 & 0.003 & 0.006 \\
0.005 & 1.002 & 0.009 & 0.010 \\
0.001 & 0.011 & 1.000 & 0.002 \\
0.000 & 0.010 & -0.006 & 0.988
\end{array}\right]
$$

This matrix is nearly the same as the identity Mueller matrix, which one would obtain in transmission for an isotropic medium. Of course, the sample is not isotropic, although its Mueller matrix appears to be that of an isotropic medium at this wavelength. The importance of the order must always be considered when analyzing these results, since a single Mueller matrix is not always sufficient to provide the characterization of a polarization element. 


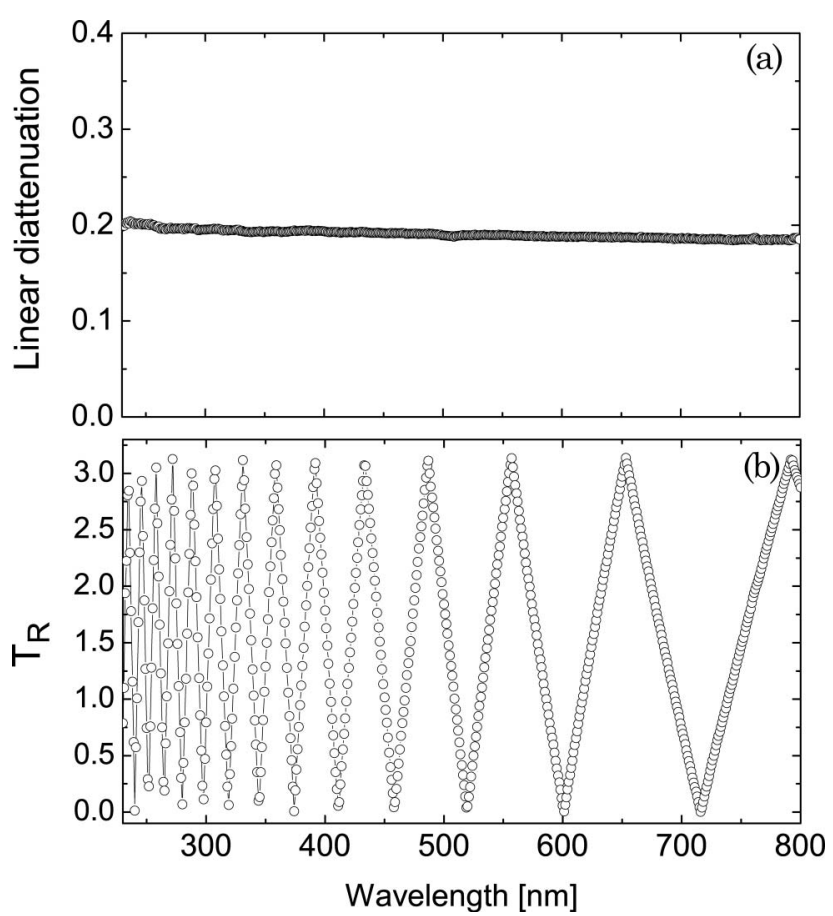

Fig. 6. (a) Linear diattenuation and (b) "folded" total retardation $T_{R}$ induced by a $1 \mathrm{~mm}$ thick, $z$-cut RH quartz plate at an incidence angle of $60^{\circ}$.

For large angles of incidence, a significant linear diattenuation appears in the measured Mueller matrices as they no longer have all the vanishing elements shown in Eq. (21). In this case, the linear diattenuation is not due to an anisotropic absorbance in quartz, but rather is caused by the different reflectivities of $s$ - and $p$-polarized light [13]. The polar decomposition allows us to obtain the linear diattenuation of the sample from the matrix factor $\mathbf{M}_{D}$ [36]. Figure 6(a) displays the linear diattenuation for a quartz plate at an angle of incidence of $60^{\circ}$.

Figure 6(b) shows the total retardance $T_{R}$ as determined using the last equation of Eqs. (24) for the RH quartz plate with an incidence angle of $60^{\circ}$. At this large incident angle, the linear birefringence of quartz is big compared to $\pi$, and the total retardance goes through several oscillations between 0 and $\pi$ as a function of wavelength. To determine the real $T_{R}$, we should also know their order to unfold the "folded" spectrum of Fig. 6. Fortunately, we do not need to know the order of the birefringence to determine the ellipticity and the gyration tensor of quartz. Equations (16) and (18) show that it is only necessary to determine the ratio of $\mathrm{CB}$ to $\mathrm{LB}$, rather than determining LB and CB separately. That is, we do not have to determine $T_{R}$. In terms of Mueller matrix elements, the ratio $\mathrm{CB} / \mathrm{LB}$ is given by

$$
\frac{\mathrm{CB}}{\mathrm{LB}}=\frac{m_{R_{12}}-m_{R_{21}}}{m_{R_{32}}-m_{R_{23}}}
$$

Therefore, the ratio $\mathrm{CB} / \mathrm{LB}$ can be straightforwardly determined once the matrix $\mathbf{M}_{R}$ is known. Although

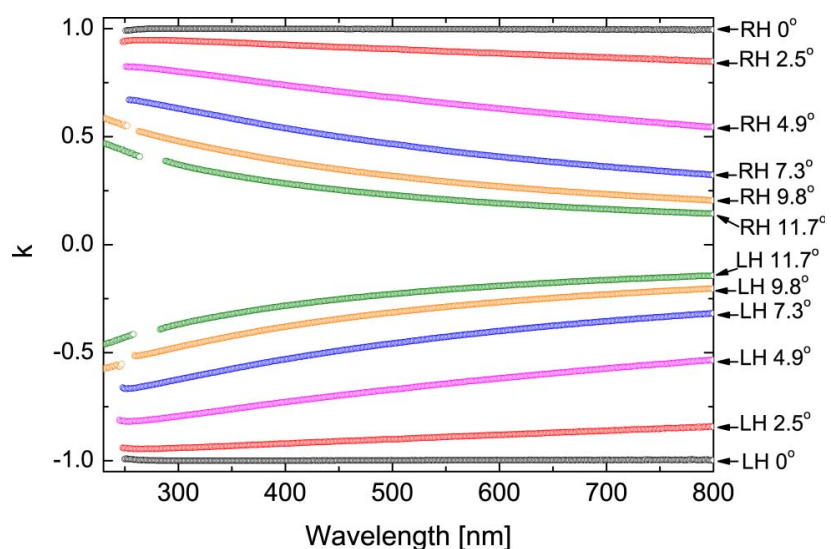

Fig. 7. (Color online) Spectroscopic evolution of the ellipticity $k$ for small angles of incidence.

Eq. (26) is a significant simplification, each of the Mueller matrix elements must be measured accurately. Obviously, a large error occurs when the denominator $\left(m_{R_{32}}-m_{R_{23}}\right)$ approaches to zero, which occurs at several wavelengths for these samples. Fortunately, as will be discussed in Section 5 , these points can be easily identified and can be removed from the calculation. Another experimental issue is that the retardation oscillation frequency increases at small wavelengths (see Fig. 6(b)), and the consecutive maxima and minima may be separated only by a few nanometers. Thus, a good spectral resolution as determined by the monochromator

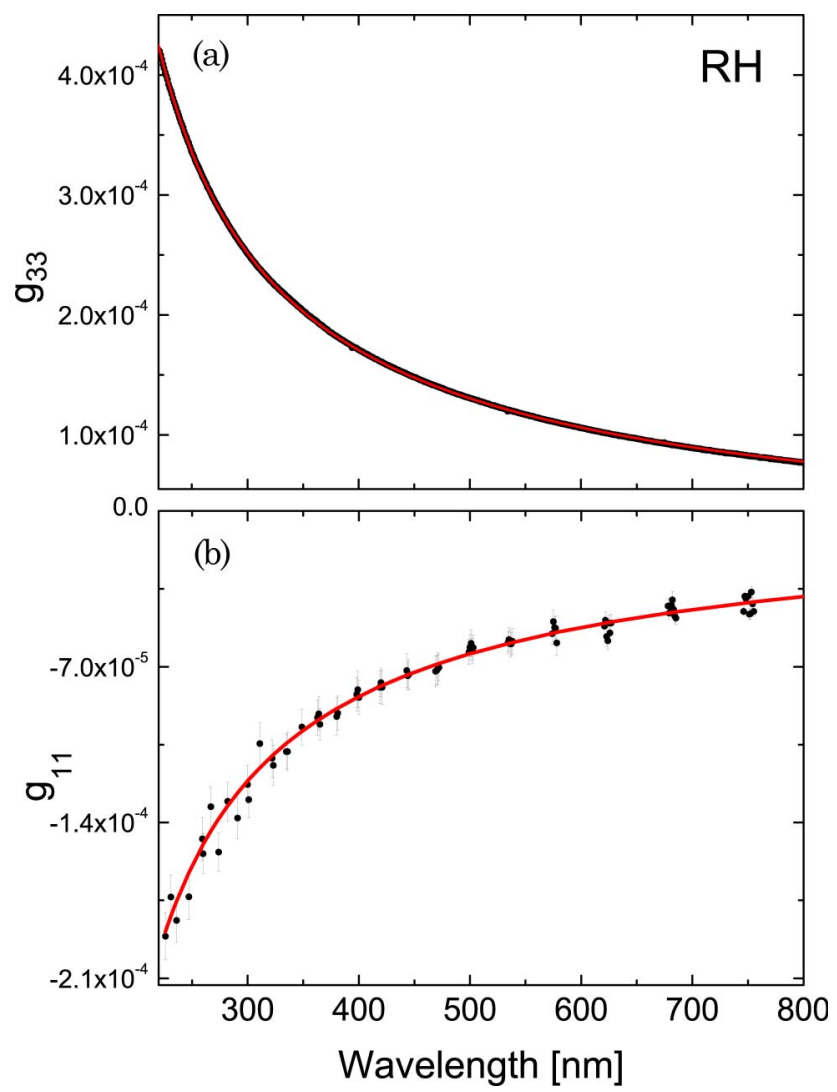

Fig. 8. (Color online) Measured components of the gyration tensor (०) and fitted dispersion relation (solid line) for the $\mathrm{RH}$ plate. 
must be sufficient to resolve these oscillations. Thinner samples would also reduce the total retardation and, therefore, avoid this problem.

\section{Results}

We have examined the retarding properties of the quartz plates at angles slightly deviated from the position of normal incidence by measuring their Mueller matrices. These measurements show the decrease on the ellipticity $k$ as we deviate from the optic axis. Figures 3 and 4, respectively, show the normalized Mueller matrix for a $\mathrm{RH}$ and a $\mathrm{LH}$ plate of quartz at small angles of incidence. Small deviations from the condition of normal incidence translate in obvious differences in the matrices, which indicate that, even for situations close to the propagation along the optical axis, the LB becomes comparable to CB. Qualitatively, these matrices resemble the matrix for a general retarder given in Eq. (21), where the different handedness is denoted by the opposite signs that Mueller matrix elements $m_{12}, m_{21}, m_{13}$, and $m_{31}$ take in Figs. 3 and 4. Elements $m_{23}$ and $m_{32}$ do not change in sign, but this is due to the geometry of our oblique incidence measurements. The only elements that are not sensitive to the handedness of quartz, no matter the orientation of the plates, are the diagonal elements.

Figure 7 shows the ellipticity $k$ for the $\mathrm{RH}$ and $\mathrm{LH}$ plates of quartz calculated from the values of LB and
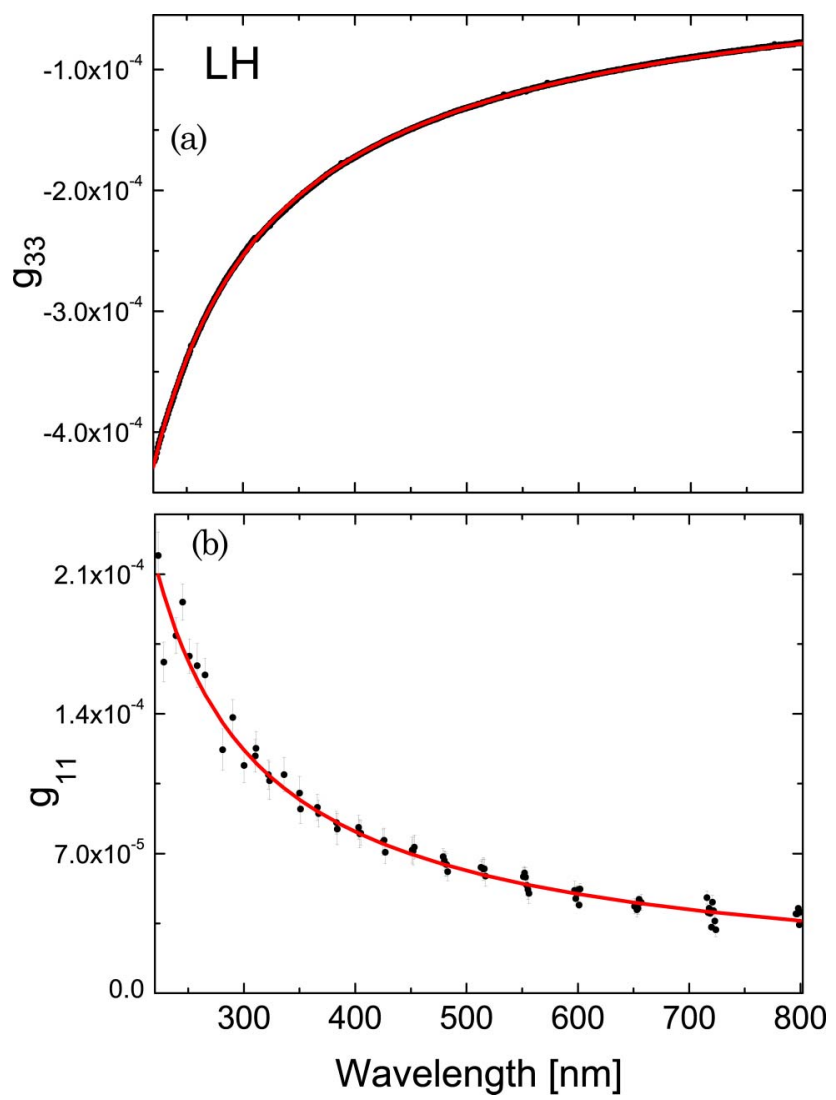

Fig. 9. (Color online) Measured components of the gyration tensor (॰) and fitted dispersion relation (solid line) for the $\mathrm{LH}$ plate.
Table 1. Fitted Parameters for the Model in Eq. (20) with $\lambda$ Expressed in Nanometers

\begin{tabular}{cll}
\hline Component & \multicolumn{1}{c}{ LH } & \multicolumn{1}{c}{ RH } \\
\hline \multirow{2}{*}{$g_{11}$} & $A_{1}=0.0277 \pm 0.0017$ & $A_{1}=-0.0298 \pm 0.0015$ \\
& $B_{1}=105.6 \pm 5.7$ & $B_{1}=91.1 \pm 4.2$ \\
& $\chi^{2}=0.82$ & $\chi^{2}=0.59$ \\
$g_{33}$ & $A_{3}=-0.0609 \pm 0.0002$ & $A_{3}=0.0604 \pm 0.0005$ \\
& $B_{3}=97.54 \pm 0.05$ & $B_{3}=97.33 \pm 0.05$ \\
& $\chi^{2}=1.33$ & $\chi^{2}=0.94$ \\
\hline
\end{tabular}

CB according to Eq. (15). The ellipticity describes the polarization state of the waves that propagate unchanged through the crystal. As expected, at normal incidence, the ellipticity is 1 for the $\mathrm{RH}$ and -1 for the $\mathrm{LH}$, and it quickly diminishes as the angle of incidence becomes more oblique. The points missing in Fig. 7 are points placed at wavelengths at which the total retardance $T_{R}$ is close to $2 \pi$, where accurate measurement of the ellipticity is not possible.

The measurement of the two independent components of the gyration tensor of quartz requires the orientation of the samples in two different configurations. The first measurement is made at normal incidence $\left(\theta=\phi=0^{\circ}\right)$, where $g_{33}$ is calculated using Eq. (14). This is the usual measurement configuration when the sample does not show LB, so the determination is straightforward. The determination of $g_{11}$ is obtained using Eq. (18) and requires that the measurements be taken at a large incidence angle to avoid small values for the $\sin \theta$ that would translate into large errors in $g_{11}$. We have chosen $\phi=$ $60^{\circ}\left(\theta \sim 33^{\circ}\right)$, which is close to the maximum incidence angle for which we can guarantee that both the extraordinary and ordinary rays are collected by the detector.

The experimental values and the fitted curves for the $g_{11}$ and $g_{33}$ components of the $\mathrm{RH}$ and the $\mathrm{LH}$ quartz plates are shown in Figs. 8 and 9. The fitting to the model of Eq. (20) has been performed with a weighted Levenberg-Marquadt nonlinear minimization procedure using the reduced $\chi^{2}$ as a figure of merit. Not all the experimental points have been used for the fitting (see Figs. 8(b) and 9(b)), since we have only used those points that correspond to wavelengths for which the ratio $\mathrm{CB} / \mathrm{LB}$ can be measured

\begin{tabular}{|c|c|c|c|}
\hline Wavelength (nm) & Reference & $\left|g_{33}\right| \times 10^{-5}$ & $\left|g_{11}\right| \times 10^{-5}$ \\
\hline \multirow[t]{6}{*}{632.8} & [11] & $10.1 \pm 0.2$ & $5.9 \pm 0.4$ \\
\hline & [10] & 10.11 & 6.11 \\
\hline & [37] & $13.6 \pm 0.5$ & $5.7 \pm 0.5$ \\
\hline & [39] & 10.528 & 5.39 \\
\hline & $\overline{[8]}$ & - & $\sim 5.2$ \\
\hline & This work ${ }^{a}$ & $10.06 \pm 0.07$ & $4.8 \pm 0.5$ \\
\hline \multirow[t]{2}{*}{510} & $\frac{[24]}{[8]}$ & $\begin{array}{c}12.96 \pm 0.2 \\
-\end{array}$ & $\begin{array}{c}5.82 \pm 0.4 \\
\sim 6.5\end{array}$ \\
\hline & This work ${ }^{a}$ & $12.81 \pm 0.08$ & $6.1 \pm 0.6$ \\
\hline
\end{tabular}

\footnotetext{
${ }^{a}$ Values obtained from the average of $\mathrm{RH}$ and $\mathrm{LH}$ results.
} 


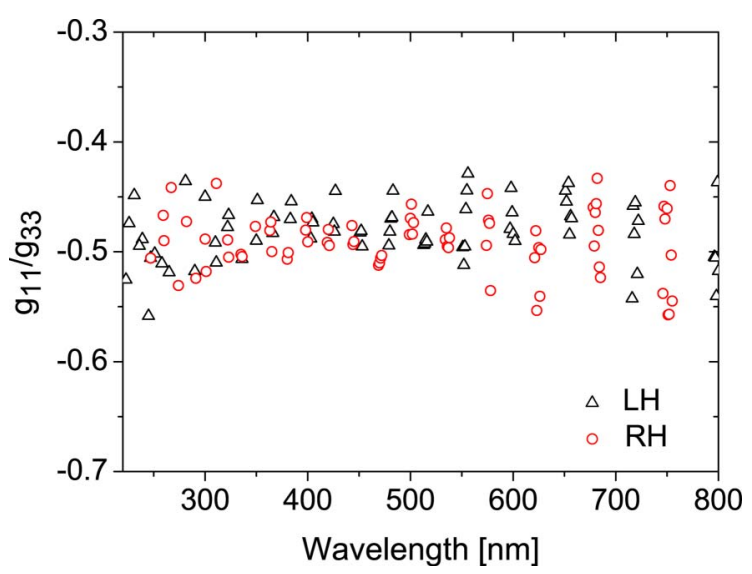

Fig. 10. (Color online) Ratio of the two independent gyration tensor components $g_{11} / g_{33}$.

with highest accuracy. In particular we have only considered points for which $\left|\mathrm{m}_{R_{32}}-\mathrm{m}_{R_{23}}\right| / 2 \geq 0.98$, i.e., points for which the absolute value of the denominator of Eq. (26) is near its maximum value.

Table 1 summarizes the fitting parameters for the four fits presented in Figs. 8 and 9 . In all cases, the dispersion relation given in Eq. (20) constitutes a good fit for experimental $g_{33}$ and $g_{11}$ data. Although the accuracy of $g_{11}$ is considerably lower than $g_{33}$, it is clear that the shape of the dispersion curves for both components are fairly similar.

A comparison between our data and some of the previously published data is presented in Table 2, at two wavelengths. There are few experimental measurements of $g_{11}$ of quartz available in the literature and most of them are restricted to a single wavelength. With the exception of Ref. [37], our results agree with literature values for $g_{33}$ within the stated error limits, and the agreement for $g_{11}$ is within 2 standard deviations with all the literature values.

We also calculated the experimental ratio of the two gyration tensor components $g_{11} / g_{33}$. In the nonabsorptive wavelength regime, this ratio should be -0.5 due to a symmetry argument that applies to the tetrahedron building units of crystalline quartz [38]. Figure 10 shows our spectroscopic experimental results for the $g_{11} / g_{33}$ ratio. They are consistent with the symmetry argument as most points are within $5 \%$ of the theoretical ideal value, and the calculated mean value is $-0.486 \pm 0.028$. By using this value in Eq. (5), we can deduce that there is no optical rotation $\overline{(G}=0)$, that is, there exists an isotropic point for the optical activity that is cylindrically symmetric around the optic axis, for propagation directed $\sim 55^{\circ}$ from the optic axis.

\section{Conclusion}

We have presented a technique for measuring the optical activity in uniaxial crystals in situations where both linear birefringence and circular birefringent effects are present. Hence, this technique allows one to measure the CB of optically active crystals in directions different from the optical axis. This technique has been applied to the measurement of the two in- dependent components of the gyration tensor of $\mathrm{RH}$ and LH crystalline quartz. The basis of the technique is the measurement of the ellipticity for the waves that transmit unchanged in their state of polarization through the crystal. For directions out of the optical axis, the ellipticity of these waves is very small and precise measurements require a highly sensitive experimental approach, such as provided by a transmission 2-MGE experiment. To our knowledge, this is the first description of a systematic methodology to obtain spectroscopic measurements of the optical activity in crystals for direction out of the optic axis.

The presented results show the ability of the 2MGE to deal with the phenomenon of optical activity in the presence of LB. Although the 2-MGE is a suitable instrument for this kind of measurement, we have presented a general approach to the measurement procedure for any instrument capable of measuring a sample Mueller matrix. We start from the basis that the Mueller matrix of the crystal can be measured, and we demonstrate how effects inherent to the optical activity can be extracted from the matrix even when much higher linear birefringence is present. Today there exist several different instruments that are capable of determining the Mueller matrix of a sample, so this method could be applied in several laboratories.

Another advantage of this technique is that different components of the gyration tensor of the crystal can be determined without having to use samples cut according to several different crystallographic planes. This is because we do not exclusively analyze light transmitted in directions perpendicular to these planes; besides, we also use a more general oblique incidence configuration in which more than one component of the gyration tensor is contributing to the observed optical activity. As an example, we have measured the $g_{11}$ and $g_{33}$ components of quartz using only a $z$-cut crystal with light incident at $60^{\circ}$ from the optic axis. In this configuration, both components $g_{11}$ and $g_{33}$ contribute to the optical activity, but, as $g_{33}$ can be well determined from normal incidence measurements, the only remaining incognita is $g_{11}$.

We envision that a similar approach to the one presented here can be used for several other crystals with point groups $3,4,6,32,422$, and 622 (international notation), which are optically active and have LB. Moreover, this technique may also be useful for crystals with point groups $\overline{4}$ and $\overline{4} \mathrm{~m}$, which do not show optical activity for light propagating along the optic axis, but do show optical rotation in directions where there is also birefringence.

O. Arteaga and A. Canillas acknowledge financial support from the Spanish government (AYA20061648-C02-01). O. A. also acknowledges financial support from the Ministerio de Educación y Ciencia (MEC) of Spain (FPU AP2006-00193). G. E. Jellison acknowledges the financial support of the Division of Materials Science and Engineering, Office of Basic Energy Sciences, U.S. Department of Energy (DOE), under contract DE-AC05-00OR22725 with Oak 
Ridge National Laboratory, managed and operated by UT-Battelle, LLC. We thank J. M. Ribó for motivating the research.

\section{References}

1. D. F. Arago, "Sur une modification remarquable qu' éprouvent les rayons lumineux dans leur passage à travers certains corps diaphanes, et sur quelques autres nouveaux phénomènes d'optique," Mem. Inst. 1, 93-134 (1811).

2. J. B. Biot, "Mémoire sur un nouveau genre d'oscillations que les molécules de la lumière éprouvent, en traversant certains cristaux," Mem. Inst. 1, 1-372 (1812).

3. T. M. Lowry, "Optical rotatory dispersion. Part I: The natural and magnetic rotatory dispersion in quartz of light in the visible region of the spectrum,” R. Soc. London Philos. Trans. A 212, 261-297 (1913).

4. C. Chou, Y.-C. Huang, and M. Chang, "Precise optical activity measurement of quartz plate by using a true phase-sensitive technique," Appl. Opt. 36, 3604-3609 (1997).

5. D. Yogev-Einot and D. Avnir, “The temperature-dependent optical activity of quartz: from le châtelier to chirality measures," Tetrahedron: Asymmetry 17, 2723-2725 (2006).

6. M. B. Myers and K. Vedam, "Effect of pressure on the optical rotatory power and dispersion of crystalline sodium chlorate," J. Opt. Soc. Am. 57, 1146-1148 (1967).

7. J. Kobayashi and Y. Uesu, "A new optical method and apparatus 'HAUP' for measuring simultaneously optical activity and birefringence of crystals. I. Principles and construction," J. Appl. Crystallogr. 16, 204-211 (1983).

8. J. R. L. Moxon, A. R. Renshaw, and I. J. Tebbutt, “The simultaneous measurement of optical activity and circular dichroism in birefringent linearly dichroic crystal sections. II. Description of apparatus and results for quartz, nickel sulphate hexahydrate and benzil," J. Phys. D 24, 1187-1192 (1991).

9. J. R. L. Moxon and A. R. Renshaw, "The simultaneous measurement of optical activity and circular dichroism in birefringent linearly dichroic crystal sections. I. Introduction and description of the method," J. Phys. Condens. Matter 2, 6807-6836 (1990).

10. C. Hernandez-Rodriguez and P. Gomez-Garrido, "Optical anisotropy of quartz in the presence of temperature-dependent multiple reflections using a high-accuracy universal polarimeter," J. Phys. D 33, 2985-2994 (2000).

11. P. Gomez and C. Hernandez, "High-accuracy universal polarimeter measurement of optical activity and birefringence of $\alpha$-quartz in the presence of multiple reflections," J. Opt. Soc. Am. B 15, 1147-1154 (1998).

12. B. Wang and T. C. Oakberg, "A new instrument for measuring both the magnitude and angle of low level linear birefringence," Rev. Sci. Instrum. 70, 3847-3854 (1999).

13. G. E. Jellison, Jr., and C. M. Rouleau, "Determination of optical birefringence by using off-axis transmission ellipsometry," Appl. Opt. 44, 3153-3159 (2005).

14. A. F. Drake, "Polarisation modulation-the measurement of linear and circular dichroism," J. Phys. E 19, 170-181 (1986).

15. L. A. Nafie, "Dual polarization modulation: a real-time, spectral-multiplex separation of circular dichroism from linear birefringence spectral intensities," Appl. Spectrosc. 54, 1634-1645 (2000).

16. G. E. Jellison, Jr., and F. A. Modine, "Two-modulator generalized ellipsometry: theory," Appl. Opt. 36, 8190-8198 (1997).

17. G. E. Jellison, Jr., and F. A. Modine, "Two-modulator generalized ellipsometry: experiment and calibration," Appl. Opt. 36, 8184-8189 (1997).

18. O. Arteaga, A. Canillas, R. Purrello, and J. Ribó, "Evidence of induced chirality in stirred solutions of supramolecular nanofibers," Opt. Lett. 34, 2177-2179 (2009).
19. G. E. Jellison, Jr., C. O. Griffiths, D. E. Holcomb, and C. M. Rouleau, "Transmission two-modulator generalized ellipsometry measurements," Appl. Opt. 41, 6555-6566 (2002).

20. G. E. Jellison, Jr., J. D. Hunn, and C. M. Rouleau, "Normalincidence generalized ellipsometry using the two-modulator generalized ellipsometry microscope," Appl. Opt. 45, 5479-5488 (2006).

21. S. Chandrasekhar, "Optical rotatory dispersion of crystals," Proc. R. Soc. A 259, 531-553 (1961).

22. G. L. Tan, M. F. Lemon, D. J. Jones, and R. H. French, "Optical properties and London dispersion interaction of amorphous and crystalline $\mathrm{SiO}_{2}$ determined by vacuum ultraviolet spectroscopy and spectroscopic ellipsometry," Phys. Rev. B 72, 205117 (2005).

23. A. Konstantinova, B. Nabatov, E. Evdishchenko, and K. Konstantinov, "Modern application packages for rigorous solution of problems of light propagation in anisotropic layered media. II. Optically active crystals," Crystallogr. Rep. (Transl. Kristallografiya) 47, 815-823 (2002).

24. G. Szivessy and C. Münster, "Über die prüfung der gitteroptik bei aktiven kristallen," Ann. Phys. 412, 703-736 (1934).

25. M. Born, Optik: Ein Lehrbuch der Elektromagnetische Lichttheorie (Springer, 1933).

26. W. Kaminsky, "Experimental and phenomenological aspects of circular birefringence and related properties in transparent crystals," Rep. Prog. Phys. 63, 1575-1640 (2000).

27. J. F. Nye, Physical Properties of Crystals: Their Representation by Tensors and Matrices (Oxford U. Press, 1985).

28. A. Konstantinova, E. Evdishchenko, and K. Imangazieva, "Manifestation of optical activity in crystals of different symmetry classes," Crystallogr. Rep. (Transl. Kristallografiya) 51, 998-1008 (2006).

29. D. Eimerl, "Quantum electrodynamics of optical activity in birefringent crystals," J. Opt. Soc. Am. B 5, 1453-1461 (1988).

30. G. Ghosh, "Dispersion-equation coefficients for the refractive index and birefringence of calcite and quartz crystals," Opt. Commun. 163, 95-102 (1999).

31. T. Bradshaw and G. H. Livens, "The formula for the optical rotatory dispersion of quartz," Proc. R. Soc. A 122, 245-250 (1929).

32. S. Chandrasekhar, "Simple model for optical activity," Am. J. Phys. 24, 503-506 (1956).

33. O. Arteaga and A. Canillas, "Pseudopolar decomposition of the Jones and Mueller-Jones exponential polarization matrices," J. Opt. Soc. Am. A 26, 783-793 (2009).

34. S.-L. Lu and A. P. Loeber, "Depolarization of white light by a birefringent crystal," J. Opt. Soc. Am. 65, 248-251 (1975).

35. R. A. Chipman, Handbook of Optics, Vol. 2: Devices, Measurements, and Properties, 2nd ed. (McGraw-Hill, 1994), Chap. 22, p. 22.31 .

36. S.-Y. Lu and R. A. Chipman, "Interpretation of Mueller matrices based on polar decomposition,” J. Opt. Soc. Am. A 13, 1106-1113 (1996).

37. J. Kobayashi, T. Asahi, S. Takahashi, and A. M. Glazer, "Evaluation of the systematic errors of polarimetric measurements: application to measurements of the gyration tensors of $\alpha$ quartz by the HAUP,” J. Appl. Crystallogr. 21, 479-484 (1988).

38. J. Jerphagnon and D. S. Chemla, "Optical activity of crystals," J. Chem. Phys. 65, 1522-1529 (1976).

39. A. Konstantinova, K. Rudoy, B. Nabatov, E. Evdishchenko, V. Stroganov, and O. Pikul', "The influence of optical activity on the intensity and polarization parameters of transmitted light in crystals," Crystallogr. Rep. (Transl. Kristallografiya) 48, 823-831 (2003). 